\title{
Impact of Offering Continuing Respiratory Care Education Credit Hours on Staff Participation in a Respiratory Care Journal Club
}

\author{
Carl R Hinkson RRT, Nirpalinder Kaur MA RRT, Michael W Sipes RRT, ${ }^{\dagger}$ \\ and David J Pierson MD FAARC
}

\begin{abstract}
BACKGROUND: Journal clubs are employed by education and healthcare institutions to facilitate learning about study design, to teach critical reading of the literature, and to help trainees and practitioners keep abreast in their fields. Our respiratory care department initiated a journal club that was open to all respiratory therapists in the community. The articles were selected by the journal club coordinator and posted on the club's web site. However, attendance remained poor despite changes in venue, time, and day of the week. In Washington State, respiratory therapists are required to obtain continuing respiratory care education credits (CRCEs), so we hypothesized that offering American Association for Respiratory Care CRCEs for journal club attendance would increase participation. METHODS: We measured journal club attendance during the 8 months preceding and the 8 months following introduction of CRCE credit for journal club attendance. The journal club meetings were held during same time frame, on the same day of the week, and in the same geographic region during the pre-CRCE and CRCE periods. Advertising for the journal club was the same during both periods as well. RESULTS: Pre-CRCE attendance ranged from 5 to 8 persons per meeting (mean \pm SD $6 \pm 1$ persons), and CRCE-period attendance ranged from 7 to 10 persons (mean \pm SD $8 \pm 1)(P=.01)$. CONCLUSIONS: Providing CRCE credits for attendance was associated with increased participation in our departmental journal club. Key words: respiratory care; journal clubs; staff education; licensure; continuing education; management. [Respir Care 2011; 56(3):303-305. @ 2011 Daedalus Enterprises]
\end{abstract}

\section{Introduction}

The journal club is a time-honored activity employed by education and healthcare institutions to facilitate learning

Carl R Hinkson RRT, Nirpalinder Kaur MA RRT, and David J Pierson MD FAARC are affiliated with the Respiratory Care Department, Harborview Medical Center, University of Washington, Seattle, Washington.

$\dagger$ Deceased

The authors have disclosed no conflicts of interest.

Carl R Hinkson RRT presented a version of this paper at the 54th International Respiratory Congress of the American Association for Respiratory Care, held December 13-16, 2008, in Anaheim, California.

Correspondence: Carl R Hinkson RRT, Respiratory Care Department, Box 359761, Harborview Medical Center, 325 Ninth Avenue, Seattle WA 98104. E-mail: gooddog@u.washington.edu.

DOI: $10.4187 /$ respcare.00952 about study design, to teach critical reading of the literature, and to help trainees and practitioners keep abreast of new developments in their fields. ${ }^{1-3}$ Journal club meetings take place at scheduled intervals, either at the workplace or off site, and typically consist of group discussions of one or more recently published articles, led by either the club's coordinator or a designated attendee. ${ }^{4,5}$ When appropriately run and tailored to attendees' educational and other needs, journal clubs increase participants' knowledge ${ }^{6,7}$ and are popular among attendees. ${ }^{8,9}$

In 2005 our institution's respiratory care department initiated a voluntary journal club to help staff members keep up with advances in the science of respiratory care as published in the peer-reviewed literature. We opened attendance to all interested respiratory therapists in the community. We posted information about the club and its meetings on the departmental web site, and linked it to the web site of our state's American Association for Respiratory Care (AARC) affiliate, the Respiratory Care Society of Washington (http://www.rcsw.org). Our journal club's 


\section{Journal Club Continuing Respiratory Care Education Credits}

meetings occurred approximately every 6 weeks. The articles and presenters were selected by the journal club coordinator $(\mathrm{CRH})$, and the articles were posted as PDF files on the web site. At each meeting an article was summarized by a preselected presenter and discussed by the whole group.

However, attendance at the journal club was poor, and remained so despite changes in venue, meeting time, and day of the week. Because Washington State requires respiratory therapists to obtain 30 continuing education credits every 2 years, with at least 10 of those being AARC continuing respiratory care education credits (CRCEs), we hypothesized that offering AARC CRCEs for journal club attendance would increase participation. After a baseline observation period, and without making other changes in format, venue, or schedule, we tested the effect on journal club attendance of providing 2 CRCEs to each participant for each meeting.

\section{Methods}

To test our hypothesis we measured journal club attendance during the 8 months preceding and the 8 months following introduction of CRCE credit for journal club attendance. The pre-CRCE period was December 2006 through July 2007, and the CRCE period was September 2007 through April 2008. Journal club meetings were held on Monday evenings, at the same time during both periods, at staff members' homes, which were in the same general area with respect to distance from the hospital during the pre-CRCE and CRCE periods. Advertising for the journal club was the same during the pre-CRCE and CRCE periods, and consisted of e-mails to staff and other potentially interested persons, flyers, and Internet postings. The administrative cost of the CRCEs was provided by the department's discretionary fund, and there was no charge to the attendees. The journal club coordinator took attendance at each meeting. To obtain CRCE credit, attendees had to read the article beforehand and pass a postmeeting test on the article.

To obtain CRCE approval for the journal club meetings we contacted the AARC and completed the application (http://www.aarc.org/education/crce_app/index.html) for a "traditional" educational activity. We also submitted an educational overview, evaluation form, presenter worksheet, and the post-meeting test for each journal club meeting. To save time and reduce costs, we sought approval for 3 meetings with each application. Each application process took about 3 hours, and at the time of the study the cost was $\$ 55$ for CRCEs for the 3 meetings.

We logged attendance data in a spreadsheet (Excel 2003, Microsoft, Redmond, Washington), and the attendance data are presented as range and mean $\pm \mathrm{SD}$. We analyzed the data with a 2-tailed Student's $t$ test. We considered a $P$ value of $<.05$ statistically significant.

\section{Results}

Journal club attendance improved after we began offering CRCEs. Pre-CRCE attendance ranged from 5 to 8 persons per meeting (mean \pm SD $6 \pm 1$ ), and CRCE-period attendance ranged from 7 to 10 persons per meeting $($ mean $\pm \mathrm{SD} 8 \pm 1)(P=.01)$

\section{Discussion}

In this small study we found an association between offering CRCEs and attendance at our departmental journal club. To our knowledge this is the first research involving a journal club that is specific to the respiratory care profession, and the first to examine the effect of offering continuing education credits on journal club attendance.

Although journal clubs are a longstanding component of continuing education activities in healthcare, poor attendance and variable attendee interest are ongoing, well documented concerns. . $^{3,9,10}$ Several authors have attempted to identify aspects of journal club structure and operation that best facilitate attendance and satisfaction, ${ }^{2,3,11,12}$ including designating a club leader, clearly defined goals, Internet marketing the club, and making the papers available to the participants beforehand.2,3

Several other aspects of journal club operation have also been touted as promoting participation, although the findings and recommendations of various authors have differed. In a review of health-professions journal clubs, Deenadayalan and colleagues found that important success factors were having regular and anticipated meetings, both long-term and short-term purposes, a trained facilitator/ leader to choose the papers and lead the discussion, established critical appraisal processes, summarizing the club's findings, and mandatory attendance. ${ }^{3}$ While our journal club has nearly all of the above features, mandating attendance in our work environment would entail paying attendees for their time, which is not an option for us. Offering CRCEs may serve as an alternative incentive.

Sidorov surveyed 131 internal medicine programs to determine factors associated with successful journal clubs. ${ }^{11} \mathrm{He}$ found that greater success was associated with smaller residency programs, mandatory attendance, having the journal club separate from the work environment, teaching formal critical appraisal skills, emphasizing original research articles rather than reviews, and providing food. Again, our journal club incorporates most of these factors.

Our journal club is not attended by our medical director or other physicians. Our intent was to emphasize discus- 


\section{Journal Club Continuing Respiratory Care Education Credits}

sion and the development of critical appraisal skills in our staff, who in some instances might be reluctant to participate if physicians were present. Although the situation is not entirely parallel, 2 studies of medical residents' journal clubs found that attendees favored having the meetings run by the residents, without faculty participation..$^{9,11}$

Other factors that have been reported to improve the quality and/or participation in healthcare journal clubs include involving a medical librarian to facilitate article selection and other aspects of the process. ${ }^{13}$ On-line journal clubs $^{10}$ and the incorporation of video conferencing into the meeting environment ${ }^{14}$ have also been reported.

Offering CRCE credits for journal club attendance has several advantages. The legitimacy and importance of the activity are emphasized, as the meetings must meet the AARC's established criteria for granting CRCE credits. Offering CRCEs also advances participating respiratory therapists toward license renewal. Forty-six states, the District of Columbia, and Puerto Rico currently require continuing education credits to maintain licensure, and the median requirement is 10 credits per year. ${ }^{15} \mathrm{~A}$ journal club offering 2 CRCEs and meeting every 6 weeks could provide 16 credits per year, so journal clubs organized similarly to ours could meet the CRCE licensure requirements.

\section{Limitations}

This was a small study with a limited sample size. Attendance data were collected from only 6 meetings prior to the intervention, and the before-and-after study design suffers from an inherent risk of bias. Although we made no changes to the journal club design or operation other than offering CRCEs, it is possible that the Hawthorne effect influenced the results. Participation in our journal club as a percentage of the total department staff remained low. At the time of this study there was approximately 80 full-time and part-time staff. At the most well attended session, 7 participants were from our institution. Although there was more participation by regular staff in the CRCE period, the majority of participants were in supervisory or leadership roles.

We cannot be certain that the improved attendance was a direct result of offering CRCEs, and other factors may have affected our findings. The pre-CRCE and CRCE periods were at different times of year (winter to summer versus fall to spring), which could have affected attendance, although one might expect attendance to fall off during the holidays (which was in the CRCE period), which would exert an effect opposite to what we found.

The articles chosen were similar in the 2 study periods, in terms of source and clinical topics. Club meetings were held on the same day and at the same time; although the staff homes hosting the meetings varied, they were of roughly equivalent distance from the hospital.

\section{Conclusions}

Providing CRCE credits was associated with increased participation in our departmental journal club. Other strategies to improve attendance that warrant exploration include developing rewards and recognitions for staff participation, and holding the session at the hospital and near shift change. Further evaluation of the journal club should focus on assessment of participants' knowledge and practice in relation to the topics discussed.

\section{REFERENCES}

1. Linzer M. The journal club and medical education: over one hundred years of unrecorded history. Postgrad Med J 1987;63(740):475-478.

2. Valentini RP, Daniels SR. The journal club. Postgrad Med J 1997; 73(856):81-85.

3. Deenadayalan Y, Grimmer-Somers K, Prior M, Kumar S. How to run an effective journal club: a systematic review. J Eval Clin Pract 2008;14(5):898-911.

4. Lee AG, Boldt HC, Golnik KC, Arnold AC, Oetting TA, Beaver HA, et al. Using the journal club to teach and assess competence in practice-based learning and improvement: a literature review and recommendation for implementation. Surv Opthalmol 2005;50:542548 .

5. Doust J, Del Mar CB, Montgomery BD, Heal C, Bidgood R, Jeacocke D, et al. EBM journal clubs in general practice. Aust Fam Physician 2008;37(1-2):54-56.

6. Linzer M, Brown JT, Frazier LM, DeLong ER, Siegel WC. Impact of a medical journal club on house-staff reading habits, knowledge, and critical appraisal skills. A randomized control trial. JAMA 1988; 260(17):2537-2541.

7. Moharari RS, Rahimi E, Najafi A, Khashayar P, Khajavi MR, Meysamie AP. Teaching critical appraisal and statistics in anesthesia journal club. QJM 2009;102(2):139-141.

8. O'Connor L, Bennett P, Gardner A, Hawkins MT, Wellman D. Implementing a journal club in a palliative care setting: a link in the chain of evidence-based practice. Collegian 2009;16(3):147-152.

9. Hartzell JD, Veerappan GR, Posley K, Shumway NM, Durning SJ. Resident run journal club: a model based on the adult learning theory. Med Teach 2009;31(4):e156-e161.

10. Lizarondo L, Kumar S, Grimmer-Somers K. Online journal clubs: an innovative approach to achieving evidence-based practice. J Allied Health 2010;39(1):e17-e22.

11. Sidorov J. How are internal medicine residency journal clubs organized, and what makes them successful? Arch Intern Med 1995; 155(11):1193-1197.

12. Jouriles NJ, Cordell WH, Martin DR, Wolfe R, Emerman CL, Avery A. Emergency medicine journal clubs. Acad Emerg Med 1996;3(9): 872-878.

13. Urquhart C, Turner J, Durbin J, Ryan J. Changes in information behavior in clinical teams after introduction of a clinical librarian service. J Med Libr Assoc 2007;95(1):14-22.

14. M'ikanatha NM, Churchill RE, Lautenbach E, Mactavish LE, Pollock TR, Weand CL, et al. Training programs to strengthen Pennsylvania's public health response. Biosecur Bioterror 2009;7(2):178186.

15. American Association for Respiratory Care. Licensure matrix. http:// www.aarc.org/advocacy/state/licensure_matrix.html\#matrix. Accessed December 23, 2010. 